\title{
Experimental Evidence of the Gardner Phase in a Granular Glass
}

\author{
A. Seguin ${ }^{1,2}$ and O. Dauchot ${ }^{3}$ \\ ${ }^{1}$ Laboratoire FAST, Université Paris-Sud, CNRS, Université Paris-Saclay, F-91405, Orsay, France \\ ${ }^{2}$ SPEC, CEA, CNRS, Université Paris-Saclay, F-91191 Gif-sur-Yvette, France \\ ${ }^{3}$ EC2M, UMR Gulliver 7083 CNRS, ESPCI ParisTech, PSL Research University, 10 rue Vauquelin, 75005 Paris, France
}

(Received 3 May 2016; published 21 November 2016)

\begin{abstract}
Analyzing the dynamics of a vibrated bidimensional packing of bidisperse granular disks below jamming, we provide evidence of a Gardner phase deep into the glass phase. To do so, we perform several compression cycles within a given realization of the same glass and show that the particles select different average vibrational positions at each cycle, while the neighborhood structure remains unchanged. The separation between the cages obtained for different compression cycles plateaus with an increasing packing fraction, while the mean square displacement steadily decreases. This phenomenology is strikingly similar to that reported in recent numerical observations when entering the Gardner phase, for a mean-field model of glass as well as for hard spheres in finite dimension. We also characterize the distribution of the cage order parameters. Here we note several differences from the numerical results, which could be attributed to activated processes and cage heterogeneities.
\end{abstract}

DOI: 10.1103/PhysRevLett.117.228001

The constituent particles of a glass are caged by their neighbors and thus cannot relax density fluctuations [1]. This is also true for hard particles under compression $[2,3]$. The associated slowing down of the dynamics is related to a complex free energy landscape with multiple glass states $[4,5]$. This picture is, however, too simple to describe the complex aging properties of glasses [6,7] as well as the observation of dynamical heterogeneities in low-temperature glasses $[8,9]$. Furthermore, when compressing hard particles to infinite pressure, a geometric transition takes place, the jamming transition, at which the dynamics is fully arrested and the particles are mechanically equilibrated [10-13]. This transition exhibits critical scalings, which characterize the marginal stability of the glass on approaching jamming and are associated with both localized and delocalized excitations [14-20]. Such features can also not be captured within the above simple landscape picture.

It was recently shown theoretically that the hard sphere glass in infinite dimension undergoes a Gardner transition [21]: glass states, envisioned as metabasins in configuration space, break up in a hierarchy of marginally stable subbasins at a low enough temperature or high enough pressure (see Fig. 1). The associated structure of the free energy landscape is necessary to capture the critical scalings of the jamming transition [22]. Later, the Gardner transition was detected numerically in a mean-field glass model [23]. The critical properties of jamming are independent of the spatial dimension [24-26], and one expects that the above findings apply in finite dimension. This was very recently confirmed in simulations of $2 \mathrm{~d}$ and $3 \mathrm{~d}$ hard sphere (HS) glasses [27]. However, a recent perturbative renormalization group study has shown that in small dimensions the transition either is governed by a nonperturbative fixed point, is first order, or simply does not exist [28]. In all cases, it remains to be observed experimentally: in practice, even if the transition does exist, finite-size and time effects, activation processes, and aging could very well hinder it.

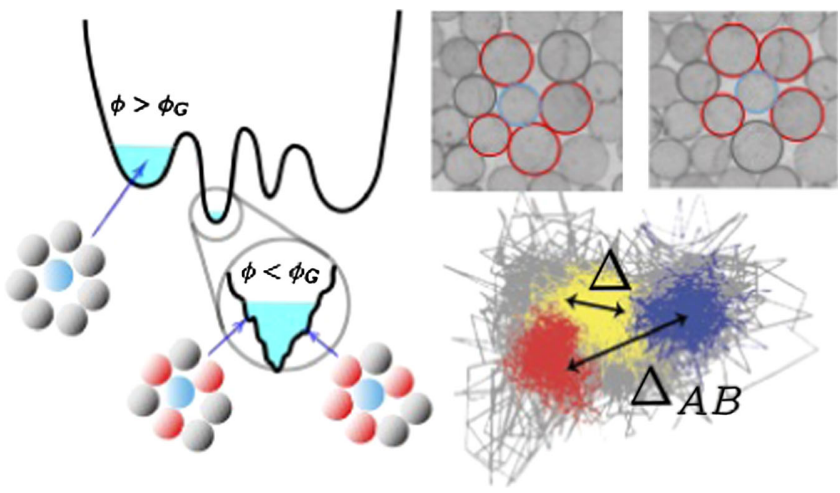

FIG. 1. Gardner transition. Left: A glass metabasin breaks into a hierarchy of marginally stable subbasins (inspired from Ref. [22]). In the glass phase $\left(\phi_{g}<\phi<\phi_{G}\right)$, the particle (in blue) is caged by its neighbors; those of which establish contact at jamming $\left(\phi_{J}\right)$ are not selected yet. In the Gardner phase $\left(\phi>\phi_{G}\right)$, each of the subbasins eventually corresponds to one structure of a contact network (red neighbors). Right top: Experimental realization of this scenario in a bidisperse system of disks for two independent compressions up to $\phi_{J}$ within the same glass state. Right bottom: In the Gardner phase, below jamming $\left(\phi_{G}<\phi<\phi_{J}\right)$, successive compressions starting from the same glass state, where particles vibrate in a large (gray) cage, lead to a different caging location in a smaller cage (blue, red, and yellow). While $\Delta(\phi)$, the cage size, decreases with $\phi, \Delta_{A B}$, the typical distance between the cages obtained for different compressions, plateaus at the cage size of the transitional packing fraction, $\phi_{G}$. 
In this Letter, we bring the first direct experimental evidence of the Gardner phase, taking advantage of a wellcontrolled granular experiment, which has already proven to successfully probe the vicinity of the jamming transition in a bidimensional granular glass former [29-35]. More precisely, following the protocol suggested for the numerical detection of the Gardner transition [23,27], taking place at $\phi_{G}$, we perform compression cycles within a given realization of the same glass and show that, for a large enough compression, the final state differs from one compression to another. To do so, we compare the average cage size within one state, $\Delta$, and the average distance separating the cages of the same particles across successive compression cycles, $\Delta_{A B}$. While for $\phi<\phi_{G}, \Delta_{A B}$ decreases like $\Delta$, it plateaus to a constant value equal to $\Delta\left(\phi_{G}\right)$, when $\phi>\phi_{G}$, signing the entrance in the Gardner phase. Despite the many nonidealities of the experimental system, the Gardner signature is remarkably strong. We also observe that, while there is no aging on the experimental time scales in the stable glass phase, it is always present in the Gardner phase as predicted theoretically. Altogether, the observed phenomenology very well matches that of the numerical observations obtained within the Mari-Kurchan (MK) mean-field model [23,36,37] and the HS systems [27]. This is all the more fascinating as the a priori important differences between a $2 \mathrm{~d}$ equilibrium hard disks and the present system of vibrated granular disks. We further characterize the fluctuations of the cage sizes $\Delta$ and intercycle cage distances $\Delta_{A B}$ and report some differences from the mean-field and HS cases. We finally discuss the possible origin of these differences as well as the importance of the Gardner phase regarding the interpretation of experimental results obtained in former studies.

Theoretical context.-By compressing a liquid and avoiding crystallization-for instance, using polydisperse systems - one ends up in a glass state. The structure is frozen and particles cannot exchange neighbors anymore. The location of the glass transition $\phi_{g}$ depends on the compression rate, and for each realization a different glass state is selected. Further compressing this glass, the pressure increases until it diverges when particles come into contact and reach mechanical equilibrium at $\phi=\phi_{J}$; the glass is jammed. In between sits the Gardner transition [21] of that glass. When $\phi>\phi_{G}$, the glass metabasin breaks into a hierarchy of marginally stable subbasins, the bottom of which corresponds to different structures of the contact network (see Fig. 1). Measuring the similarity of the contact network between jammed structures obtained after independent compressions is one way of testing the existence of the marginal phase [22]. Alternatively, one can follow the cage dynamics $[23,27]$. Since the detection of contacts in experiments is prone to some arbitrariness, we will follow this second path.

Starting from a glass state at a packing fraction $\phi_{0}>\phi_{g}$ and realizing independent compressions up to a packing fraction $\phi$, one should follow particle trajectories $\mathbf{r}_{i}^{k}(t)$ in the final state $\mathrm{k}$ and compute (i) for each compression the mean square displacement (MSD) $\Delta^{k}\left(t, t_{w}\right)$ and (ii) for each pair of compressions, the "mean square distance" $\Delta^{k k^{\prime}}(t)$ between the two compressed states $\left(k, k^{\prime}\right)$ :

$$
\begin{gathered}
\Delta^{k}\left(t, t_{w}\right)=\frac{1}{N} \sum_{i=1}^{N}\left|\mathbf{r}_{i}^{k}\left(t_{w}+t\right)-\mathbf{r}_{i}^{k}\left(t_{w}\right)\right|^{2}, \\
\Delta^{k, k^{\prime}}(t)=\frac{1}{N} \sum_{i=1}^{N}\left|\mathbf{r}_{i}^{k}(t)-\mathbf{r}_{i}^{k^{\prime}}(t)\right|^{2} .
\end{gathered}
$$

Averaging over compressions and thermal samples, one obtains $\Delta\left(t, t_{w}\right)=\overline{\left\langle\Delta^{k}\left(t, t_{w}\right)\right\rangle}$ and $\Delta_{A B}(t)=\overline{\left\langle\Delta^{k, k^{\prime}}(t)\right\rangle}$. For $\phi<\phi_{G}$, if the glass is well equilibrated (i.e., at large enough $\left.t_{w}\right), \Delta_{A B}(\forall t)=\lim _{t \rightarrow \infty} \Delta\left(t, t_{w}\right)$. On the contrary, for $\phi>\phi_{G}$, equilibrium is never reached and $\Delta_{A B}(t)$ remains larger than $\Delta\left(t, t_{w}\right)$, even at large $t_{w}$. The large $t_{w}$ behavior of $\delta \Delta\left(t, t_{w}\right)=\Delta_{A B}\left(t+t_{w}\right)-\Delta\left(t, t_{w}\right)$ is thus a good dynamical order parameter of the Gardner transition. One sees, however, that long time limits have to be considered and that aging in the Gardner phase significantly complicates the analysis (see $[23,27]$ for a more complete discussion). In finite dimension, the situation is even less clear. The cages are heterogeneous, and activated dynamics will prevent the configurations at $\phi_{0}$ from constraining the dynamics at arbitrarily long times, so that the glass itself is never fully equilibrated. Moving to experiments, one has to deal with finite time issues, as in simulations, but also with specifically experimental constraints such as limited spatial resolution and possible artifacts such as slow but persistent convection currents [38].

Experimental implementation.-The experimental setup already described elsewhere [29] consists in a monolayer of 8500 bidisperse (44\% large, $56 \%$ small) photoelastic disks of stiffness $\kappa=1660 \mathrm{~N} \cdot \mathrm{m}^{-1}$, and friction coefficient $\mu \simeq 0.4$, with diameters $d_{s}=4 / 5 d_{l}=4 \pm 0.01 \mathrm{~mm}$. The disks are laid out on a horizontal glass plate vibrated horizontally with frequency $f=10 \mathrm{~Hz}$ and amplitude $A=10 \mathrm{~mm}$ and confined in a cell fixed in the laboratory frame. The packing fraction $\phi$ can be varied by tiny amounts $\left(\delta \phi / \phi \sim 5 \times 10^{-4}\right)$. The stroboscopic motion of a set of 1600 grains in the center of the sample is tracked by a CCD camera synchronized with the plate. The position of the grains is detected with an accuracy of $10^{-2} d_{s}$. In the following, lengths are measured in $d_{s}$ units and time in cycle units.

Starting from a low packing fraction $\phi$, we gradually compress the system until it reaches a highly jammed state following the same protocol as in Ref. [38]. Then we stepwise decrease the volume fraction until $\phi_{J}$, where (i) the pressure measured at the wall in the absence of vibration falls to zero and (ii) the contacts observed through 
cross-polarizers disappear, signaling the unjamming transition. The precise value of $\phi_{J}$ varies with each realization of the preparation protocol. This realization specific value is here $\phi_{J}=0.8236 \pm 0.0003$. Then we gently decompress the system further down to $\phi_{0}=0.8185$, where we check that most of the disks keep the same neighbors (less than $3 \%$ neighbor exchange during the entire experiment), ensuring that the system remains in the same glass state. Furthermore, we shall compute all the relevant quantities on the subset of particles that were never lost during the image processing or tracking procedures and have never lost neighbors. We then perform ten sets of compression cycles, from $\phi_{0}$ to $\phi \in\left[\phi_{0}, \phi_{J}\right]$, each cycle containing ten compressions. The compression cycles are rapid quenches separated by $10^{3}$ vibration cycles.

Vibrated granular matter is prone to develop convection as soon as it unjams [38]. These convective currents develop over length scales of tens of particle diameters. The best way to concentrate on the vibrational dynamics and eliminate spurious effects is to analyze the dynamics of each particle in the reference frame of the center of mass of its neighbors. Doing so, we possibly remove other possible interesting effects, including spatial correlations. We however stick to this strategy, which ensures that we focus on the vibrational properties and guarantees not to attribute the Gardner transition to any other effects. Also, we shall see below that we kept some of the interesting phenomenology of dynamical heterogeneities. This technique has the advantage of not subtracting an overall convection field, which, locally, can be large as compared to the intrinsic displacement. We thus redefine the particle position as $\mathbf{r}_{i} \rightarrow \mathbf{r}_{i}-\mathbf{r}_{i}^{\Omega}$, where $\mathbf{r}_{i}^{\Omega}=1 / n_{i} \sum_{j} \mathbf{r}_{j}$, where the sum runs over the neighbors of particle $i$ and $n_{i}$ is the number of them. We are then in a position to compute $\Delta^{k}\left(t, t_{w}\right)$ and $\Delta^{k k^{\prime}}(t)$ as defined by Eqs. (1) and (2). We average over compressions and replace the average over samples by temporal averaging to obtain $\Delta(t)$ and $\Delta_{A B}$. Doing so, we assume aging to be negligible on the 200 time steps time window used here (see below the discussion about aging).

Figure 2 displays $\Delta=\Delta\left(t_{0}=500\right)$ and $\Delta_{A B}$ as a function of $\phi$. It is the first and main experimental result of the present study. The typical MSD within cages $\Delta(\phi)$ steadily decreases with the packing fraction. By contrast, $\Delta_{A B}(\phi)$ first decreases like $\Delta(\phi)$ but then plateaus close to $10^{-2}$ for $\phi \gtrsim 0.820$. This demonstrates that there is a regime at large $\phi$, before jamming, in which several cage configurations are separated by an average distance larger than the cage size. Also, $\Delta$ alone presents no sign of a transition suggesting that the glass metabasin is broken in subbasins with distributed cage sizes rather than in subbasins with a well-defined smaller cage size. The system has entered the Gardner phase at $\phi_{G} \simeq 0.820$. The numerical values of $\phi_{J}$ and $\phi_{G}$ are realization specific. Still, the relative distance of the Gardner transition to jamming, $\phi_{G} / \phi_{J} \simeq 0.996$, provides a naive estimate of the pressure at the Gardner

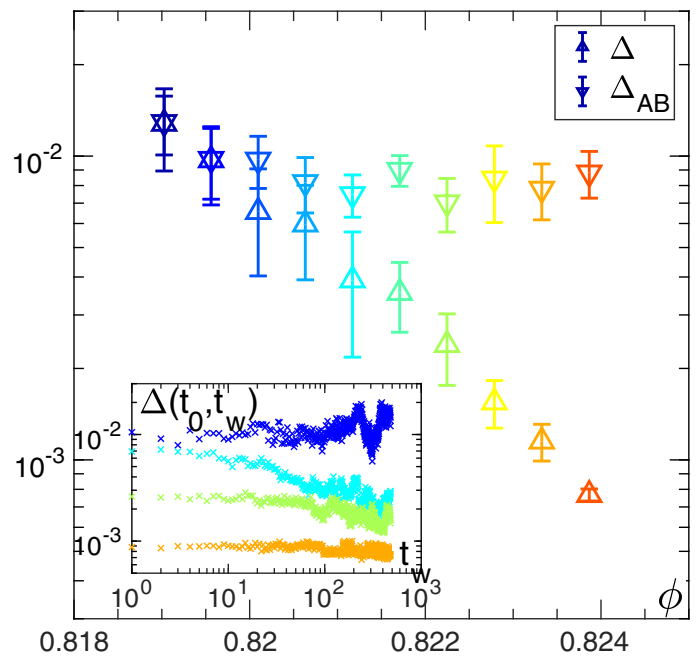

FIG. 2. Cage order parameters. Mean square displacement $\Delta\left(t_{0}\right)$ and mean square distance between cages $\Delta_{A B}$ averaged over 200 time steps and ten compression cycles from $\phi_{0}=$ 0.8185 to $\phi$ as a function of $\phi$. Inset: Aging. Mean square displacement $\Delta\left(t_{0}, t_{w}\right)$ as a function of $t_{w}$ for $\phi=0.8196$, $0.8212,0.8222,0.8233$. The color codes are for the packing fraction.

transition $p_{G} \simeq 1 /\left(\phi_{J}-\phi_{G}\right) \simeq 3 \times 10^{2}$, which allows us to locate the present realization of the Gardner transition in the phase diagram of $2 \mathrm{~d}$ hard disks [23,27]. Very remarkably, although the present system is frictional and the jamming packing fractions are lower than for thermal hard disks, our observations are consistent with the numerical estimates. Extrapolating $\Delta$ to $10^{-1}$, the typical MSD values reported for the experimental and numerical glass transition, we find $\phi_{g}=0.815$, which is also consistent with previously reported values for the glass transition in $2 \mathrm{~d}$ systems of bidisperse hard grains $[39,40]$.

The inset in Fig. 2 displays the aging of $\Delta\left(t_{0}, t_{w}\right)$ for a set of four packing fractions $\phi$. For the lowest packing fraction (top curve) $\phi<\phi_{G}, \Delta\left(t_{0}, t_{w}\right)$ remains constant: there is no aging, as it should be if the glass is stable. The three larger packing fractions are in the Gardner phase. We observe aging, which corresponds to the slow drift of the system among the marginal Gardner states. Note that the aging is slower for larger packing fractions, hence the fact that for the densest packing (bottom curve) it is hardly noticeable on the experimental time scale. The observation of aging above $\phi_{G}$ further confirms that the phase observed at a high packing fraction is truly Gardner.

We now turn to the analysis of the fluctuations of $\Delta$ and $\Delta_{A B}$. Remember that we deal with a single glass sample. Hence, fluctuations have only two possible sources: temporal fluctuations inside each compression cycle and intercycle fluctuations. The cage size fluctuations across time and cycles, normalized by the mean cage size [Fig. 3(a)], present large exponential tails. This observation strongly contrasts with that reported in the numerical study 


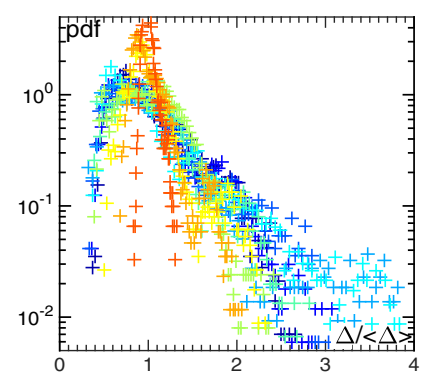

(a)

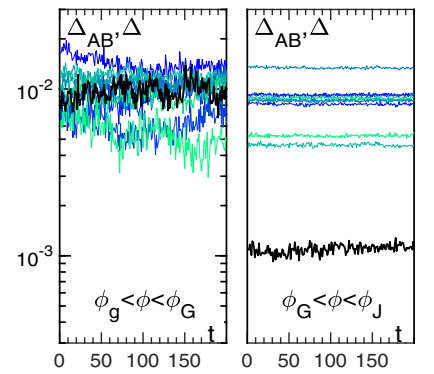

(c)

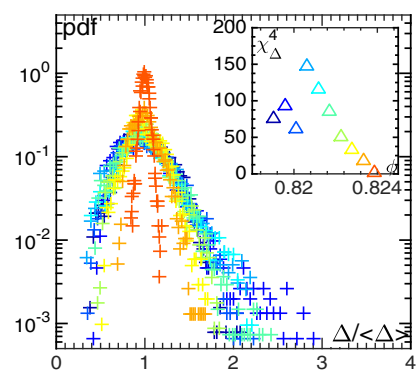

(b)

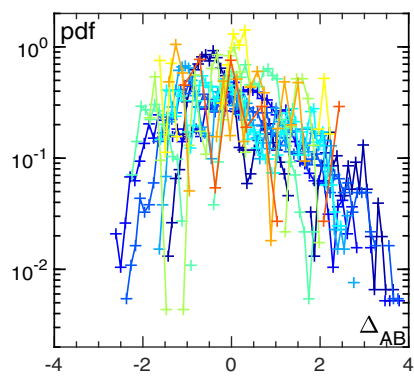

(e)
FIG. 3. Statistics of $\Delta$ and $\Delta_{A B}$. Probability distribution of $\Delta /\langle\Delta\rangle$ sampled (a) over time and compression cycles and (b) over time only [inset: dynamical susceptibility associated to the fluctuations of $\Delta^{k}\left(t_{0}, t_{w}\right)$ over time]. Color code as in Fig. 2. Temporal evolution of $\Delta^{k, k^{\prime}}(t)$ for ten different pairs of compression cycles (color coded) for (c) $\phi=0.8196$ and (d) $\phi=0.8228$, as compared to the temporal evolution of $\Delta^{k}\left(t, \tau_{0}\right)$ (in black). Colors indicate different pairs of compression cycles. (e) Probability distribution function of $\Delta_{A B}$ sampled across compression cycles. Color code as in Fig. 2.

of the MK mean-field model [23]. In that case, the distributions for $\Delta$ are Gaussian-like at all $\phi$. Figure 3(b) shows the distribution for the same quantity but sampled separately within each cycle. They exhibit similar exponential tails, which account for both the aging drift and the intrinsic temporal fluctuations of $\Delta^{k}\left(t_{0}, t_{w}\right)$. Such fluctuations highlight the presence of dynamical heterogeneities already reported for the same system $[29,34]$ as well as in numerical simulations of harmonic spheres [41]. Those heterogeneities can be quantified by computing in each cycle, and then averaging over the cycles, the associated dynamical susceptibly $\chi_{\Delta}^{4}\left(t_{0}\right)=\mathrm{N} \operatorname{Var}\left[\Delta\left(t_{0}, t_{w}\right) / \sigma\right]$, where $\sigma$ is the standard deviation of the cage size for a single particle. This intrastate dynamical susceptibility presents a maximum around the Gardner transition [inset in Fig. 3(b)]. Although this susceptibility is not computed in Ref. [27], the distributions of $\Delta$, there also, present signs of nonGaussianity at $\phi_{G}$. This remarkable feature, absent from mean-field results, is an interesting target for future theoretical developments in finite dimension.

The fluctuations of $\Delta_{A B}$ are better captured by following the temporal evolution of $\Delta^{k, k^{\prime}}(t)$ for different pairs of compression cycles [Figs. 3(c) and 3(d)]. When $\phi<\phi_{G}$, $\Delta^{k, k^{\prime}}(t)$ fluctuates around the same value as $\Delta^{k}\left(t_{0}, t_{w}\right)$, and the fluctuations overlap from one compression cycle to another: the system explores the whole metabasin of the glass. By contrast, when $\phi>\phi_{G}$, the distances between different pairs of compressed states are very different and much larger than the typical cage size. In the numerical studies in Refs. [23,27], the authors report that the distributions for $\Delta_{A B}$ sampled over many samples, starting from a Gaussian-like distribution for $\phi<\phi_{G}$, develop exponential tails near $\phi_{G}$, before broadening again at larger $\phi$. As a result, the skewness of the $\Delta_{A B}$ distributions exhibits a maximum around $\phi_{G}$. This skewness is attributed to sample to sample fluctuations, which are absent in the present experimental study. We have access only to the distributions of the reduced centered distribution of $\Delta_{A B}$ sampled over the pairs of cycles. Despite the strong lack of statistics [Fig. 3(e)], a systematic positive skewness is present here too.

Discussion and perspectives.-We have conducted the first direct experimental observation of the Gardner phase, deep into the glass phase, before jamming. Although it has been obtained in a rather specific type of - granular-glass, there is now much evidence that such glasses are good models for hard potential thermal glass formers [42]. At the level of the average order parameters of the transition, we obtain an excellent agreement with the results obtained in the numerical studies of the MK model [23] and hard sphere glasses [27]. We observe some differences in the fluctuations statistics of the cage size; in particular, our results suggest that intrastate dynamical heterogeneities are maximal at the Gardner transition. Further quantitative comparisons with existing numerical simulations require significantly more work, which are far beyond the scope of the present study, from both the numerical and the experimental side. The importance of aging, the role of activated processes, and the finite size effects should be quantified precisely. To do so, studying the dynamics of the caging processes is obviously an important and promising next step.

The present study sheds new light on several results obtained previously in the same experimental setup. In particular, it would be very interesting to investigate how the large dynamical heterogeneities observed just below jamming $[29,30,34,38]$ are related to the marginality of the many glass states in the Gardner phase. Another striking observation, which must contain a signature of the Gardner phase, is the avalanche dynamics observed in the motion of an intruder pulled through the glass, at constant force or constant velocity $[32,43]$. Finally, the elasticity and dilatancy softening, reported in Refs. [33,44,45], might have close connections to the breakdown of classical elasticity reported for an amorphous solid in the Gardner phase [46-49].

We thank Francesco Zamponi, Patrick Charboneau, and Giulio Biroli for many interesting discussions related to this work and Yuliang Jin for assisting us in defining the compression protocols using preliminary simulations of our system. We also thank Vincent Padilla and Cécile 
Wiertel-Gasquet for invaluable technical support. Part of this work was supported by Paris Sciences et Lettres Grant JAM-T\&E and Investissements d'Avenir LabEx Physique: Atomes Lumière Matière (ANR-10-LABX-0039-PALM).

[1] A. Cavagna, Phys. Rep. 476, 51 (2009).

[2] F. Krzakala and J. Kurchan, Phys. Rev. E 76, 021122 (2007).

[3] M. Mézard and G. Parisi, in Structural Glasses and Supercooled Liquids (Wiley, Hoboken, NJ, 2012), pp. 151-191.

[4] M. Goldstein, J. Chem. Phys. 51, 3728 (1969).

[5] P. G. Debenedetti and F. H. Stillinger, Nature (London) 410, 259 (2001).

[6] M. Goldstein, J. Non-Cryst. Solids 357, 249 (2011).

[7] D. Fragiadakis and C. M. Roland, Phys. Rev. E 86, 020501 (2012).

[8] K. Vollmayr-Lee, W. Kob, K. Binder, and A. Zippelius, J. Chem. Phys. 116, 5158 (2002).

[9] P. Ballesta, A. Duri, and L. Cipelletti, Nat. Phys. 4, 550 (2008).

[10] C. S. O’Hern, L. E. Silbert, A. J. Liu, and S. R. Nagel, Phys. Rev. E 68, 011306 (2003).

[11] G. Parisi and F. Zamponi, Rev. Mod. Phys. 82, 789 (2010).

[12] M. van Hecke, J. Phys. Condens. Matter 22, 033101 (2010).

[13] A. J. Liu, S. R. Nagel, W. van Saarloos, and M. Wyart, The Jamming Scenario-An Introduction and Outlook (Oxford University Press, New York, 2011).

[14] M. Wyart, L. E. Silbert, S. R. Nagel, and T. A. Witten, Phys. Rev. E 72, 051306 (2005).

[15] C. Brito and M. Wyart, J. Stat. Mech., (2007)L08003,

[16] C. Brito and M. Wyart, J. Chem. Phys. 131, 024504 (2009).

[17] K. Chen, W. G. Ellenbroek, Z. Zhang, D. T. N. Chen, P. J. Yunker, S. Henkes, C. Brito, O. Dauchot, W. van Saarloos, A. J. Liu, and A. G. Yodh, Phys. Rev. Lett. 105, 025501 (2010).

[18] E. Lerner, G. During, and M. Wyart, Soft Matter 9, 8252 (2013).

[19] E. DeGiuli, E. Lerner, C. Brito, and M. Wyart, Proc. Natl. Acad. Sci. U.S.A. 111, 17054 (2014).

[20] P. Charbonneau, E. I. Corwin, G. Parisi, and F. Zamponi, Phys. Rev. Lett. 114, 125504 (2015).

[21] E. Gardner, Nucl. Phys. B257, 747 (1985).

[22] P. Charbonneau, J. Kurchan, G. Parisi, P. Urbani, and F. Zamponi, Nat. Commun. 5, 3725 (2014).

[23] P. Charbonneau, Y. Jin, G. Parisi, C. Rainone, B. Seoane, and F. Zamponi, Phys. Rev. E 92, 012316 (2015).

[24] M. Skoge, A. Donev, F. Stillinger, and S. Torquato, Phys. Rev. E 74, 041127 (2006).
[25] C. P. Goodrich, A. J. Liu, and S. R. Nagel, Phys. Rev. Lett. 109, 095704 (2012).

[26] P. Charbonneau, E. I. Corwin, G. Parisi, and F. Zamponi, Phys. Rev. Lett. 109, 205501 (2012).

[27] L. Berthier, P. Charbonneau, Y. Jin, G. Parisi, B. Seoane, and F. Zamponi, Proc. Natl. Acad. Sci. U.S.A. 113, 8397 (2016).

[28] P. Urbani and G. Biroli, Phys. Rev. B 91, 100202 (2015).

[29] F. Lechenault, O. Dauchot, G. Biroli, and J.-P. Bouchaud, Europhys. Lett. 83, 46003 (2008).

[30] F. Lechenault, R. Candelier, O. Dauchot, J.-P. Bouchaud, and G. Biroli, Soft Matter 6, 3059 (2010).

[31] C. Brito, O. Dauchot, G. Biroli, and J.-P. Bouchaud, Soft Matter 6, 3013 (2010).

[32] R. Candelier and O. Dauchot, Phys. Rev. Lett. 103, 128001 (2009)

[33] C. Coulais, A. Seguin, and O. Dauchot, Phys. Rev. Lett. 113, 198001 (2014).

[34] C. Coulais, R. P. Behringer, and O. Dauchot, Europhys. Lett. 100, 44005 (2012).

[35] L. Berthier, P. Chaudhuri, C. Coulais, O. Dauchot, and P. Sollich, Phys. Rev. Lett. 106, 120601 (2011).

[36] R. Mari, F. Krzakala, and J. Kurchan, Phys. Rev. Lett. 103, 025701 (2009).

[37] R. Mari and J. Kurchan, J. Chem. Phys. 135, 124504 (2011).

[38] C. Coulais, R. P. Behringer, and O. Dauchot, Soft Matter 10, 1519 (2014).

[39] A. R. Abate and D. J. Durian, Phys. Rev. E 74, 031308 (2006).

[40] R. Candelier, A. Widmer-Cooper, J. K. Kummerfeld, O. Dauchot, G. Biroli, P. Harrowell, and D. R. Reichman, Phys. Rev. Lett. 105, 135702 (2010).

[41] A. Ikeda, L. Berthier, and G. Biroli, J. Chem. Phys. 138, 12A507 (2013).

[42] O. Dauchot, in Glasses and Grains, edited by B. Duplantier, T. C. Halsey, and V. Rivasseau (Springer, Basel, 2011), pp. 137-157.

[43] R. Candelier and O. Dauchot, Phys. Rev. E 81, 011304 (2010).

[44] M. Otsuki and H. Hayakawa, Phys. Rev. E 90, 042202 (2014).

[45] B. P. Tighe, Granular Matter 16, 203 (2014).

[46] E. Lerner, E. DeGiuli, G. Duering, and M. Wyart, Soft Matter 10, 5085 (2014).

[47] D. Nakayama, H. Yoshino, and F. Zamponi, arXiv: 1512.06544v1 [J. Stat. (to be published)].

[48] A. K. Dubey, I. Procaccia, C. A. B. Z. Shor, and M. Singh, Phys. Rev. Lett. 116, 085502 (2016).

[49] G. Biroli and P. Urbani, arXiv:1601.06724v1. 